\title{
Measuring and Evaluating the Differences of Compared Images for a Correct Car Silhouette Categorization using Integral Transforms
}

\author{
Elena Pivarčiová ${ }^{1}$, Daynier Rolando Delgado Sobrino², Yury Rafailovich Nikitin ${ }^{3}$, Radovan Holubek², \\ Roman Ružarovský \\ ${ }^{1}$ Department of Manufacturing and Automation Technology, Faculty of Environmental and Manufacturing Technology, \\ Technical University in Zvolen, Masarykova 24, 96001 Zvolen. \\ ${ }^{2}$ Institute of Production Technologies, Faculty of Materials Science and Technology in Trnava, Slovak University of \\ Technology in Bratislava, Jána Bottu street, 25, 917 24, Trnava, Slovak Republic. \\ ${ }^{3}$ Department of Mechatronic Systems, Faculty of Quality Management, Kalashnikov Izhevsk State Technical University, \\ Studencheskaya street, 7, 426069, Izhevsk, Russia.
}

\begin{abstract}
The present paper focuses on the analysis of the possibilities of using integral transforms for measuring and evaluating the differences of compared images (car silhouettes) with the purpose of a correct car body categorization. Approaches such as the light intensities frequency change, the application of discrete integral transforms without the use of further supplementary information enabling automated data processing using the Fourier-Mellin transforms are used within this work. The calculation of the several metrics was verified through different combinations that implied using and not using the Hamming window and a low-pass filter. The paper introduced a method for measuring and evaluating the differences in the compared images (car silhouettes). The proposed method relies on the fact that the integral transforms have their own transformants in the case of translation, scaling and rotation, in the frequency area. Besides, the Fourier-Mellin transform was to offer image transformation that is resistant to the translation, rotation and scale.
\end{abstract}

Keywords: Automatic measurement system, Fourier-Mellin transform, image analysis, Body in White (BIW), car silhouettes.

\section{INTRODUCTION}

Even when it has been long known that perceived quality is one of the most important factors underlying the success of car manufacturers today [1], sometimes some of the more conventional methods used for quality control in the automotive industry are regarded as outdated and ineffective to a certain extent. Thus, given this importance of product control in the manufacturing process and the challenging nature of ensuring that the quality of each product meets the standards required by the clients in an efficient and effective manner, the combined use of such conventional (manual) and new automated methods of control have been gaining more and more popularity in recent years and significant research has been focused on the topic.

In the middle of all these needs for quality control and measurements, automatic computer vision has particularly and increasingly become an important tool in the measurement for the evaluation of quality and in the recognition of several types of effects in the automotive industry, see the relevant works appearing in [2] for a better understanding of this. The main goal of a vision measurement system lies in trying to emulate, to a given extent, some of the human vision, sense, image and scene evaluation capabilities, and this by electronic and informatics means involving tasks as recognition, digitalization and evaluation, among others. If, for example, a measured input is sufficiently accurate and does not require specific difficult processing of input data (images), the system can autonomously react to it and good results are reachable relatively easily. On the contrary, if the same system were to be implemented into a real environment, and its inputs were not images with clearly and distinctly defined objects of a constant size, rate and observation angle, the whole process would not be so easy and those images would require significant pre-processing to get the proper reaction from the system. Similarly, the accuracy of measurements also depends on a delicate process of offline calibration, and in some practical applications, the system is even supposed to be reconfigured on an almost continuous basis so as to track the target that an online calibration requires [3]. In the end, the main goal of all this reflects and enhances the need of building an automatic measurement 
system for quality control in the automotive industry without the requirements of constant or frequent human interference. This proposal should be also general and wide enough so that it can be used in other branches of manufacturing.

Automated visual measuring is known to be an imageprocessing method that has been widely applied over the years in the production line for quality control purposes [4], [5]. This focuses on mechanical parts, vehicles, casting production and even the garment industry [6], [7]. Inspection tasks, however, are time consuming and have been usually and mostly carried out through human intervention, i.e.: human inspectors. On the other hand, the inspectors' performance has been often a subject of debate in the scientific community and practice, making some authors state and prove to an extent that this is often inadequate and its accuracy is influenced by the fatigue of the intense and repetitive tasks. In addition, human inspectors need training which together with the needed skills implies investments and time to develop. Also, if compared to machines, the hours of human inspectors are relatively limited, and labor costs are a major factor posing a challenge for most manufacturers trying to compete in a densely populated and competitive marketplace [8]. On the other hand, an automatic measurement system needs almost no human presence and thus, eventually, this translates into more time available to the experts to better and more effectively work in the measurement and to analyze the composite image data increasing the speed and effectiveness of their work [9].

Some of the key advantages of computer machine vision measurement systems lie in the use of contactless nondestructive measuring [10], the use of ultraviolet and infrared radiation for the exploring and inspection tasks, as well as in the augmented flexibility allowing for immediate changes of system configuration and the possibility of parallel measuring of more parameters at the same time. These types of systems are applied in many areas of society, for example: in traffic, medicine, manipulators and robotics [11], [12], assembly cells, production of synchronous DC motors [13], welding [14] and of course, in quality inspection.

In this study, as it is in principle similarly stated in [15], an automatic measurement system is understood and seen as an optical system with image detection and verification which is to be used for the detection and proper categorization (classification and sorting) of car silhouettes. One of the most problematic issues for the automatic measuring system relates to the comparison of the recorded images with a reference template, i.e.: car silhouette template comparison [16]. Besides, the system also needs to possess the ability to detect whether the given casting is faultless or defective. This last task is relatively easy for the human eye and mind, because humans have experience which has been in many cases cumulated since birth. However, this same problem is difficult to process for a computer, getting computers to fully understand things and the effects of the surrounding world has been a myth for long and still is a present challenge for science.

During the car production/assembly phase, it is highly necessary to monitor and control the production process to detect potential errors of different types and nature and, among other things, to also sort the car bodies by specific models and distribute them according to their type and the place where such parts are required, see Fig.1. One of the key parts of the process is the one related to the creation of the Body in White (BIW) assemblies. In the process of their obtaining, all models of cars are welded on a single line. This welding line almost always contains samples of welded car bodies of all models which are to be compared with the ones being produced every single time. Here an automatic visual measuring system plays a decisive role in the quality of the car bodies, and robots are mainly the ones responsible for "seeing, comparing, and controlling that these match what the ideal car of a given model should look like, in other words: they are to compare the model of a given product with its equivalent product being manufactured at a given time.

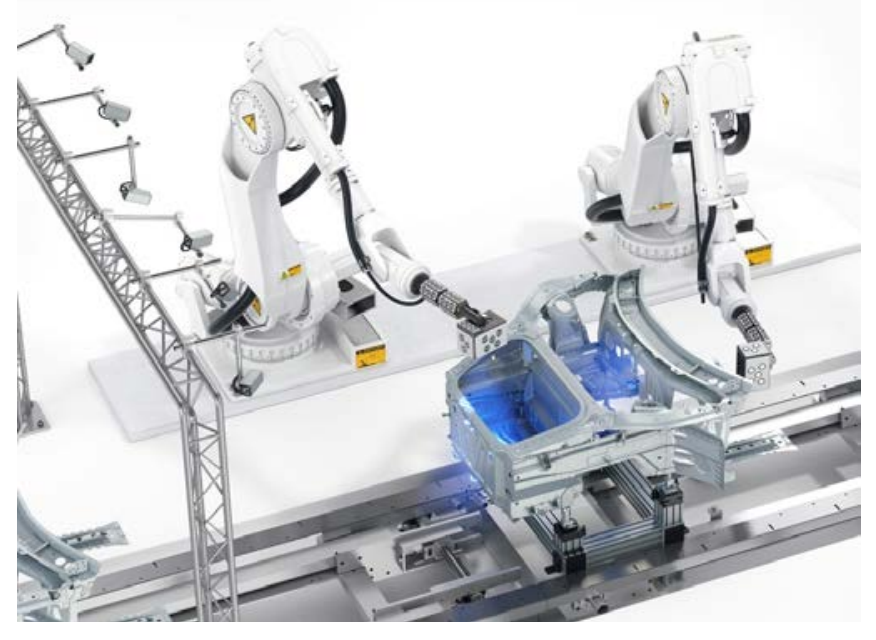

Fig.1. 3D inline measuring technology from ZEISS [17].

Another possible application for this type of automated system is found in the distribution of the BIW to the respective assembly halls/lines, in other words, in the sorting of these according to individual car body types.

Taking into consideration all the above-mentioned elements, in this paper we propose a method that compares the reference image with the tested image using the amplitude and phase spectra of the Fourier-Mellin transform, also their invariability to translation, rotation and scale. Given that the Fourier-Mellin transform reduces the mutual rotation of the images along the horizontal axis and the shift in scaling along the vertical axis, then any two images, one of which may offset, rotated and/or with a change in scale, have identical Fourier-Mellin transform amplitudes. For comparison purposes and/or the determination of the level of similarity, we used in the paper several metrics, for example the phase correlation and percentage comparison analysis.

\section{SUBJECT \& METHODS}

A phase-only correlation technique/method that is based on Fourier-Mellin transform was used in the automatic measurement system for the purposes of image analysis and comparison. 


\section{A. The basics of the Fourier-Mellin transform}

The Fourier-Mellin transform allows comparing images which are offset, rotated and have changed scale. This method takes advantage of the fact that the shift differences are annulated because the amplitude spectrum of the image and its displaced copy are identical, only their phase spectrum is different. Subsequently the log-polar transform causes the rotation and scale to appear outwardly as a shift. On the other hand, phase correlation is based on the fact that two similar images in a cross-spectrum create a continuous sharp extreme in the place of registration and the noise is distributed randomly in discontinuous extremes.

This is the reference image $a(x, y)$ and tested image $b(x, y)$ that happens to be the copy of the image $a(x, y)$ and is rotated, translated and has changed scale.

In the case of the Fourier transform of images (1), it applies:

$$
\begin{aligned}
& \mathrm{A}(u, v)=\mathrm{F}\{a(x, y)\}=|\mathrm{A}(u, v)| \mathrm{e}^{-\mathrm{j} \theta(u, v)} \\
& \mathrm{B}(u, v)=\mathrm{F}\{b(x, y)\}=|\mathrm{B}(u, v)| \mathrm{e}^{-\mathrm{j} \theta(u, v)}
\end{aligned}
$$

where $|\mathrm{A}(u, v)|,|\mathrm{B}(u, v)|$ are the spectral amplitude, $\theta(u, v)$ is the phase, $\mathrm{F}\{$. $\}$ is the Fourier transform [16].

On the other hand, the Fourier transform of log-polar amplitudes is equivalent to the Fourier-Mellin transform [18]. Next, we detail the following cases:

a) The log-polar transform of amplitudes $|A(u, v)|,|B(u, v)|$ from the Cartesian coordinate system to the log-polar coordinate system.

The Fourier transform is displayed next in a log-polar plane as indicated by the transformation of coordinates, Fig.2.

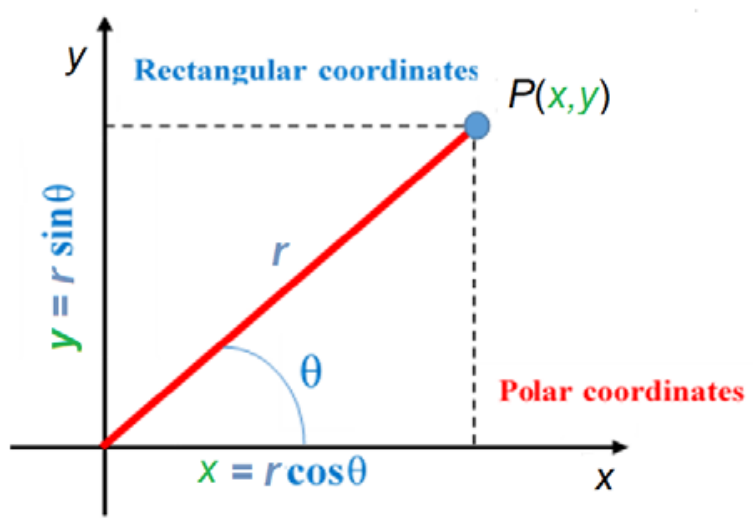

Fig.2. Transform of rectangular to polar coordinates.

In order to convert from Cartesian to log-polar coordinates we do as stated in (2):

$$
r=\sqrt{\mathrm{x}^{2}+\mathrm{y}^{2}}
$$

The beginning of the log-polar coordinate system $\left(m_{0}, n_{0}\right)$ should be in the middle of the video matrix, and this in order to ensure the maximum number of pixels possible.
The maximum sampling radius for the conversion will be defined as in (3):

$$
\begin{aligned}
& \rho_{\max }=\min \left(m_{0}, n_{0}\right) \ldots \text { inscribed circle } \\
& \rho_{\text {max }}=\min \left(m_{0}, n_{0}\right) \ldots \text { inscribed circle }
\end{aligned}
$$

The relationship between the polar coordinates $(\rho, \theta)$, used for the samples of the test image, and the log-polar image $\left(e^{r}, \theta\right)$ is explained by $(4)$ :

$$
(\rho, \theta)=\left(e^{\mathrm{r}}, \theta\right)
$$

For mapping the pixels of the test image $\left(x_{\mathrm{i}}, y_{\mathrm{i}}\right)$ to the pixels of the output image $\left(r_{\mathrm{m}}, \theta_{\mathrm{n}}\right)(5)$ was used, see [19], where $\left(\rho_{m}, \theta_{n}\right)=\left(e^{r_{m}}, \theta_{n}\right)$

$$
\begin{gathered}
x_{\mathrm{i}}=\operatorname{round}\left(\rho_{\mathrm{m}} \cdot \cos \theta_{\mathrm{n}}+m_{0}\right) \\
y_{\mathrm{j}}=\operatorname{round}\left(\rho_{m} \cdot \sin \theta_{\mathrm{n}}+n_{0}\right)
\end{gathered}
$$

b) Fourier transform of log-polar amplitudes (6).

$$
\begin{aligned}
& \boldsymbol{A}_{\text {ip }}(\boldsymbol{v}, \boldsymbol{\omega})=F\left\{\mid A_{\text {ip }}\left(e^{\mathrm{r}}, \theta \mid\right\}\right. \\
& \boldsymbol{B}_{\text {ip }}(\boldsymbol{v}, \boldsymbol{\omega})=F\left\{\mid B_{i \mathrm{p}}\left(e^{\mathrm{r}}, \theta \mid\right\}\right.
\end{aligned}
$$

In this case the log-polar transform of the amplitude spectrum causes the rotation and scale to be shown as a translation, these can be better understood from the compound figure that appears below, where the amplitude and the phase spectrum of the Fourier transform of the real image of the car part appears in $a$ ), and its Fourier spectrum in log-polar coordinates is shown in $d$ ).
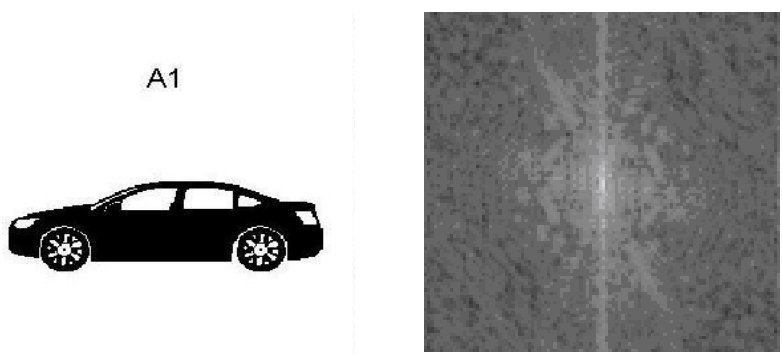

a)

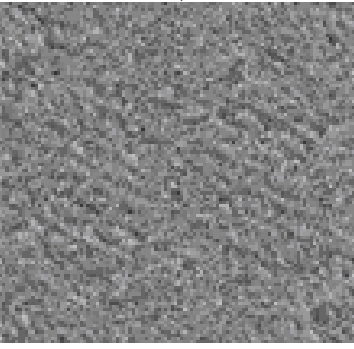

c) b)

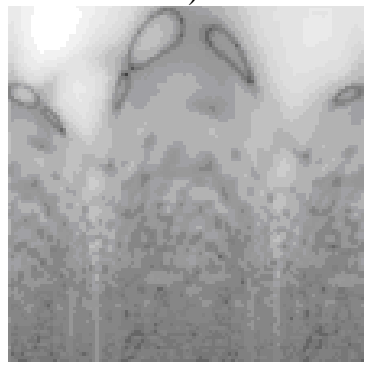

d)
Fig.3. The amplitude and the phase spectrum of the Fourier transform: a) Original image, b) Fourier spectrum amplitude in the Cartesian coordinates, c) Fourier spectrum phase in the Cartesian coordinates, d) Fourier spectrum in log-polar coordinates. 


\section{B. Comparison of the observed images of measured objects} and their mutual interaction

In general terms the use of the Fourier-Mellin transform reduces the mutual rotation of images to translation along the horizontal axis, and also the change of scale to vertical axis translation [20]. Both of these cases/spectra were compared. These authors analyzed two images, one of which was moved and/or rotated and/or had the scale changed, and they proved that both ended up having identical amplitude of FourierMellin transform. Based on this study, reference designs were compared in our case with test images in order to determine the degree of similarity and correlation between them for the purposes of our automatic measurement system.

Besides, several well-known and other more specific metrics were also used for the calculation of a comparative score helping to evaluate the results of the comparison of the obtained images. This score/metrics quantifies the similarities between the test image and the reference image. The calculation of the metrics has been verified in different combinations, and this with or without the application of the Hamming window, and similarly with or without the application of the low-pass filter. The representative metrics are used to determine the similarity between reference and test images. These metrics are detailed next.

\section{POC, Phase-Only Correlation}

This comparative metric is calculated as the maximum phase correlation according to (7), where $F$ stands for Fourier transform of images $a, b, F^{-1}$ is the inverse Fourier transform, and $F^{*}$ is the complex conjugate image [20].

$$
\mathrm{C}=F^{-1}\left(\frac{F_{a} F_{b}{ }^{*}}{\left|F_{\mathrm{a}}\right|\left|F_{\mathrm{b}}\right|}\right)
$$

The phase correlation gives a distinct maximum if two images are similar. On the contrary, a more insignificant maximum will be created if two images are not similar. The size of the maxima is used as a measure of similarity between two images.

\section{MPOC, Modified Phase-Only Correlation}

Since the energy of the signal is lower in the high-frequency domain, phase components are not reliable in high-frequency ones [16]. The effect of unreliable phase components in high frequencies can be limited by using filters or modifying the POC function by using the spectral weighting function.

The function of spectral weighting $W(u, v)$ has been used to improve the detection by removing minor ingredients with high frequency, which have a low reliability, where $\mathrm{u}, \mathrm{v}$ are $2 \mathrm{D}$ coordinates, $\beta$ is a parameter, which checks the width of the function, and $\alpha$ is used only for normalizing purposes (8) [21].

$$
W(u, v)=\left(\frac{u^{2}+v^{2}}{\alpha}\right) e^{-\frac{u^{2}+v^{2}}{2 \beta^{2}}}
$$

Such a modified image phase correlation function of $\boldsymbol{a}$ and $\boldsymbol{b}$ appearing in (9) is given by [27].

$$
\tilde{q}_{a, b}(x, y)=F^{-1}\left\{W(u, v) \frac{F_{a}(u, v) F_{b}^{*}(u, v)}{\left|F_{a}(u, v)\right|\left|F_{b}(u, v)\right|}\right\}
$$

The extreme value of a function $\tilde{q}_{a, b}(x, y)$ is invariable at the change of shift and brightness. In our case, this value was used to measure the similarity of images: if two images are similar, their function MPOC gives a crisp extreme, if they are different, then the extreme decreases considerably.

\section{PD, Percent Discrimination}

This metric has relative amount of similarity between reference and test image as seen in (10) [22].

$$
\mathrm{PD}=\frac{2\left[C_{a b}\right]_{\max }}{\left[C_{a a}\right]_{\max }+\left[C_{b b}\right]_{\max }} x 100 \%
$$

where: $\left[C_{a a}\right]_{\max },\left[C_{a b}\right]_{\max }$, and $\left[C_{b b}\right]_{\max }$ are maximal phase correlations if they are compared to a reference image $a(x, y)$ with itself, tested image $b(x, y)$ with itself, and the reference image $a(x, y)$ with the image $b(x, y)$.

These metrics were used to compare the log-polar amplitudes of Fourier-Mellin transform of images.

\section{A functional testing of the proposed method}

Designs of car body silhouettes available over the internet have been used as an example for the functional testing of the proposed method, see Fig.4. For the image comparison purposes, 3 basic car silhouette samples were considered, and 10 images were used for each case what yielded a total of 30 . Besides, in order to obtain better and more reliable results, a series of extra comparison tests were conducted where each image was compared with the others and itself, what in the end generated a total of 90 tested images. It is important to underline that 30 of these were comparison of correct (right) pairs and the remaining 60 of incorrect ones.
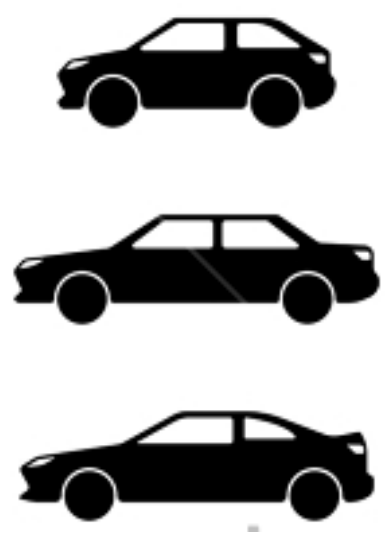

Fig.4. The design of car bodies, Stock Vector Illustration. Silhouette cars on a white background.

The before-mentioned metrics Maximum phase correlation (POC, Phase Only Correlation), Modified phase correlation (MPOC, Modified Phase Only Correlation), Percentage comparison (PD, Percent Discrimination)) were used to determine the similarity of the reference and test images - 
calculation of the comparison score. The calculated score was compared with the threshold value, which determines the degree of correlation necessary for the comparison to be taken as a match.

The different threshold values $t$ regulating the decisionmaking process were set based on several performed tests:

- images generating a result higher than $t$ are evaluated as identical or highly similar, i.e.: it can be stated it is the same car body,

- images generating results lower than or equal to $t$ are regarded to be inconsistent, i.e.: it cannot be stated it is the same car body.

The possible decisions resulting from the system are of the type: match, mismatch, and no consistent result at all. It is however possible to find varying degrees of strong matches and mismatches.

The number of correctly rejected and correctly accepted images depends on the threshold value defined, see Fig.5. The value is adjustable depending on the requirements of the process, what may make the system more or less accurate in some cases. As the test results showed, the thresholds values $t$ of the relevant criteria was set as follows:

•threshold values I. $\quad \bullet$ minimum of the correct pairs

-threshold values II. $\quad$ maximum of the wrong pairs

- selected threshold value $t \bullet$ maximum of the threshold

- it's not the same car body values I. and II.

- score $\leq$ threshold value

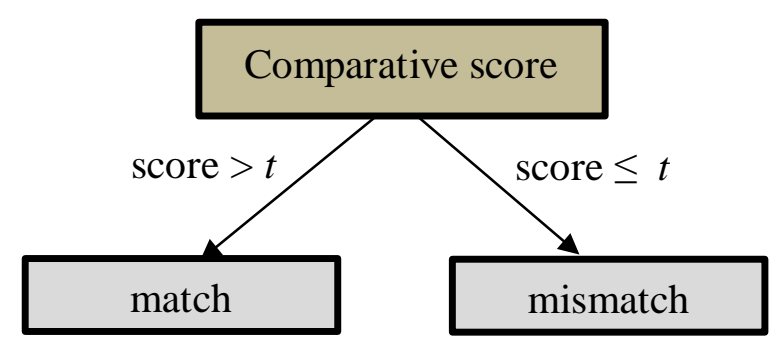

Fig.5. Comparative score issuing a match and/or mismatch of images.

\section{RESULTS}

In our particular case, the images were adjusted to 256 shades of grey and to the dimensions of $256 \times 256$ pixels for obtaining faster results with the Fourier transform. The test images with the parameters for the transformation were created from the basic/primary images. The images were translated in the horizontal and/or vertical direction, rotated around the different angles in both directions and also had the scales changed. The resulting images were compared with other car bodies.

Using the proposed method, the system identified 93 percent of correct products. On the other hand, only the remaining 7 percent of the products that were identified by the system to be correct (red color in graph), happened to be incorrect instead, and this was based on the analysis of the images as described in our method, see Fig.6. for a better understanding of the results. The results proved the detection method used was valid, and thus it could be used and become a significant asset in the classification, sorting and inclusion of car bodies into the relevant categories (categorization).

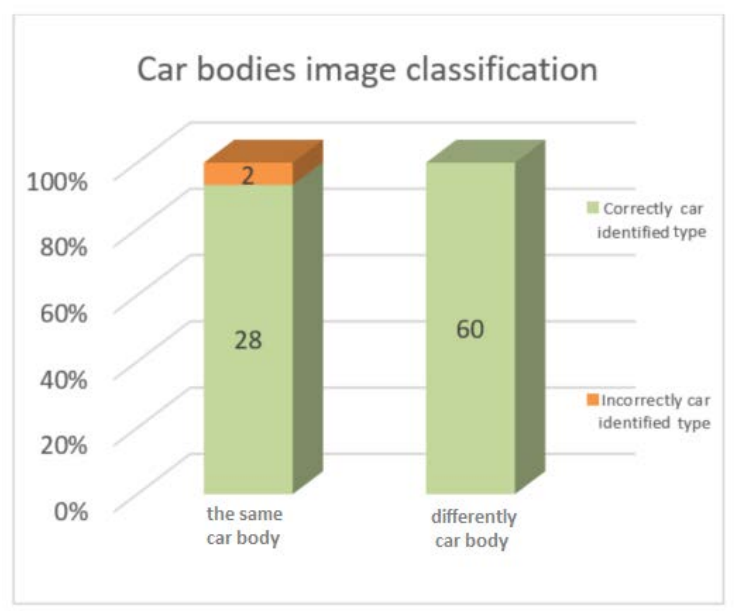

Fig.6. Results of the tested images.

The high success rate was achieved due to the use of a comparative set with rather small angle of rotation and scaling. The maximum correct recognition limit of this method has not been tested yet. Similarly, images have not been properly recognized in the case of the scale being too changed ( 0.7 and 1.3$)$, and/or in combination with translation and rotation.

\section{CONCLUSION}

As mentioned before, the measurement process is a key part of any kind of production, what is especially important in the case of the automotive industry. One of the main outputs of any measurement process is the achievement of high levels of quality of the products. This represents the main premise for the company success and its positioning among the competitors. Besides, this all fully depends on the use of new technologies, automation and implementation of methods into the related manufacturing processes, modified based on [23], [24].

The verification of quality by automatic measurement systems provides an even higher chance to compete in the related field as well as the level of quality checks allows its further improvement. In the course of the manufacturing process or in the product completion various defects can occur, as it is stated in the ISO/TS16949, which is technical specification aimed at the development of a quality management system that provides for continual improvement, emphasizing defect prevention and the reduction of variation and waste in the supply chain.

The calculated metrics were compared to the verification threshold that determined the degree of correlation necessary for the comparison, issuing what was to be taken as match. Based on tests carried out, the threshold values of $\boldsymbol{t}$ were set, according to which the system decision is always regulated: images generating the result greater than or equal to $\boldsymbol{t}$ are evaluated as identical, in other words: It is the same car body 
part; on the other hand, images generating results lower than $\boldsymbol{t}$ are evaluated as non-compliant, i.e.: these are not the same car body parts.

The decisions of system can be: match, mismatch, and without result, even though there are possible changing degrees of strong matches and mismatches.

The number of properly rejected and properly accepted images depends on the present threshold value of $\boldsymbol{t}$. The value is adjustable depending on the requirements, thus the system could be more or less accurate depending on this. In other words, it all depends on each particular case study and the levels of confidence and requirements needed for the verifications. In the case of the automotive industry and our case in particular, these values tend to be of $100 \%$, i.e.: the threshold should be established so as to have no errors in the process of detection.

\section{CONCLUSIONS}

The evolution of computing technology is progressing hand in hand with the evolution of other natural, technical or economic sciences. Adequate powerful computing technology enables high precision analysis of process models that more precisely describe the physical or social nature of things [25].

The paper describes the design of a method for the correct car body classification (car silhouettes) based on the principles of Fourier-Mellin transform and subsequent comparison and analysis of images using Fourier transform and phase correlation. The Fourier-Mellin transform proved to offer image transformation resistant to translation, rotation and scale. The proposed method uses the fact that integral transforms have their transformants in the case of translation, scaling and rotation in the frequency domain.

With the use of the automatic measurement system it was possible to compare silhouettes of car bodies and find out if these belonged to the same type or not. This was supported by the use of several key criteria for the matches, such as the phase correlation, the difference correlation, the correlation coefficient, the percentage comparison and the comparison of calculated values with the chosen threshold for the relevant criterion. In the frame of the experiments, threshold values have been set in a way that all the wrong couples of body works can be revealed.

\section{ACKNOWLEDGMENT}

This publication is the result of implementation of the project "University Scientific Park: Campus Mtf Stu - CAMBO” (ITMS: 26220220179) supported by the Research and Development Operational Program funded by the EFRR and by project KEGA MŠ SR 003TU Z-4/2016: Research and education laboratory for robotics and RFBR research project № 18-08-00772 A.

\section{REFERENCES}

[1] Stylidis, K., Wickman, C., Söderberg, R. (2015). Defining perceived quality in the automotive industry: An engineering approach. Procedia CIRP, 36 (2015), 165-170.
[2] Bogue, R. (2013). Robotic vision boosts automotive industry quality and productivity. Industrial Robot: An International Journal, 40 (5), 415-419.

[3] Di Leo, G., Liguori, C., Pietrosanto, A., Sommella, P. (2017). A vision system for the online quality monitoring of industrial manufacturing. Optics and Lasers in Engineering, 89, 162-168.

[4] Ružarovský, R., Delgado Sobrino, D.R., Holubek, R., Koštál, P. (2014). Automated in-process inspection method in the flexible production system iCIM 3000. Applied Mechanics and Materials, 693, 50-55.

[5] Božek, P., Pivarčiová, E. (2013). Flexible manufacturing system with automatic control of product quality. Strojarstvo, 55 (3), 211-221.

[6] Mery, D., Jaeger, T., Filbert, D. (2002). A review of methods for automated recognition of casting defects. http://www.academia.edu/20111824/A_review_of_me thods_for_automated_recognition_of_casting_defects.

[7] Świłło, S.J., Perzyk, M. (2013). Surface casting defects inspection using vision system and neural network techniques. Archives of Foundry Engineering, 13 (4).

[8] Dhillon, B.S. (2009). Human Reliability, Error, and Human Factors in Engineering Maintenance. CRC Press.

[9] Huang, S.-H., Pan, Y-Ch. (2015). Automated visual inspection in the semiconductor industry: A survey. Computers in Industry, 66, 1-10.

[10] Frankovský, P., Ostertag, O., Trebuňa, F., Ostertagová, E., Kelemen, M. (2016). Methodology of contact stress analysis of gearwheel by means of experimental photoelasticity. Applied Optics, 55 (18), 4856-4864.

[11] Kováč, J., Ďurovský, F., Hajduk, M. (2014). Utilization of virtual reality connected with robotized system. Applied Mechanics and Materials, 613, 273-278.

[12] Frankovský, P., Hroncová, D., Delyová, I., Hudák, P. (2012). Inverse and forward dynamic analysis of two link manipulator. Procedia Engineering, 48, 158-163.

[13] Abramov, I.V., Nikitin, Yu.R., Abramov, A.I., Sosnovich, E.V., Božek, P. (2014). Control and diagnostic model of brushless DC motor. Journal of Electrical Engineering, 65 (5), 277- 282.

[14] Jena, D.B., Kuma, R. (2011). Implementation of wavelet denoising and image morphology on welding image for estimating HAZ and welding defect. Measurement Science Review, 11, (4).

[15] Neogi, N. Mohanta, K.D., Dutta, K.P. (2014). Review of vision-based steel surface inspection systems. EURASIP Journal on Image and Video Processing, 2014 (50).

[16] Ito, K., Nakajima, H., Kobayashi, K., Aoki, T., Higuchi, T. (2004). A fingerprint matching algorithm using Phase-Only Correlation. IEICE Transactions on Fundamentals of Electronics, Communications and Computer Sciences, E87-A (3), 682-691.

[17] Carl Zeiss Ltd. (2018). 3D inline measuring technology from ZEISS. https://www.zeiss.co.uk.

[18] Druckmüller, M., Antoš, M., Druckmüllerová, H. (2005). Mathematical methods for visualization of the solar corona. Jemná mechanika a optika, 10, 302-304. 
[19] van den Dool, R. (2004). Fourier and Mellin Transform. Image Processing Tools. www.scribd.com/doc/9480198/Tools-Fourier-MellinTransform.

[20] Derrode, S., Ghorbel, F. (2001). Robust and efficient Fourier-Mellin transform approximations for graylevel image reconstruction and complete invariant description. Computer Vision and Image Understanding, 83 (1), 57-78.

[21] Gueham, M., Bouridane, A., Crookes, D. (2007). Automatic recognition of partial shoeprints based on phase-only correlation. In IEEE International Conference on Image Processing. IEEE, Vol. 4, 441444.

[22] Chen, Q.S. (1993). Image registration and its applications in medical imaging. Dissertation work, Vrije University, Brussels, Belgium.
[23] Slížik, J., Hart’anský, R. (2012). Metrology of electromagnetic intensity measurement in near field. Quality Innovation Prosperity, 17 (1), 57-66.

[24] Hallon, J., Kováč, K., Bittera, M. (2018). Comparison of coupling networks for EFT Pulses Injection. Przeglad elektrotechniczny, 94 (2), 17-20.

[25] Hart'anský, R., Smieško, V., Rafaj, M. (2017). Modifying and accelerating the method of moments calculation. Computing and Informatics, 36 (3), 664682.

Received April 17, 2018 Accepted July 18, 2018 